\title{
Modèle hydro-écologique du lac de Tunis
}

\author{
A. Ben Slimane $\left({ }^{*}\right)$, L. Masbernat $\left({ }^{* *}\right)$, M. Moussa $\left({ }^{*}\right)$
}

\section{Introduction}

Le lac de Tunis (fig. I) a fait l'objet, durant les années 1986-88, d'importants travaux d'aménagements en vue de la restauration de la qualité de ses eaux et la création d'une nouvelle ville de 300000 habitants sur ses berges. Une campagne de mesure couvrant d'une part, l'année 1984 et d'autre part, les années 1988-89 a été réalisée afin de suivre l'évolution de la qualité des eaux du lac, avant et après les travaux.

Un modèle hydro-écologique du lac de Tunis a été développé afin de prévoir l'évolution de la qualité des eaux du lac après les aménagements réalisés. Ce modèle est composé d'un modèle physique (hydrodynamique, salinité et température) couplé à un modèle de qualité des eaux.

\section{Modèle physique}

Le modèle physique prédétermine le champ de vitesse, de température et de salinité. Des essais préalables en utilisant un modèle $2 \mathrm{D}$ de ces variables intégrées sur la profondeur (Réf. [1] et [2]) ont mis en évidence (fig. 2 et 3) le caractère unidirectionnel des écoulements dans le lac après implantation de la digue et gestions des échanges à la passe de Keredine.

On a donc adopté pour la modélisation des variables physiques un modèle $1 \mathrm{D}$ basé sur les équations suivantes, où est adoptée l'hypothèse de l'onde diffusante pour le modèle hydrodynamique et où l'on a négligé les phénomènes dispersifs longitudinaux (instationnarités lentes) :

$$
\begin{gathered}
\frac{\partial A}{\partial t}+\frac{\partial Q}{\partial X}=E_{h} \\
\frac{\partial Q}{\partial t}=-g A \frac{\partial\left(Z_{f}+h\right)}{\partial X}+\frac{1}{\rho}\left(\tau_{i}-\tau_{p}\right) \\
\frac{\partial S}{\partial t}+\frac{\partial(Q S)}{\partial X}=E_{s} \\
\frac{\partial T}{\partial t}+\frac{\partial(D T)}{\partial X}=\frac{1}{h} \frac{\Phi}{\rho C_{p}}+E_{T} .
\end{gathered}
$$

(*) ENIT, Dép. Hydraulique, BP 37, Le Belvédère, 1002, Tunis, Tunisie.

(**) IMFT, LA 005-CNRS, avenue du Pr Camille Soula, 31400, Toulouse, France.

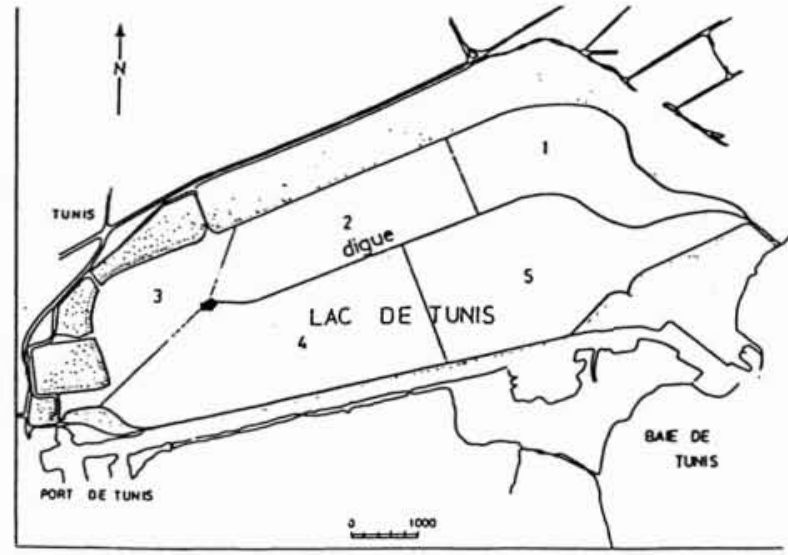

1. Le lac de Tunis après aménagements.

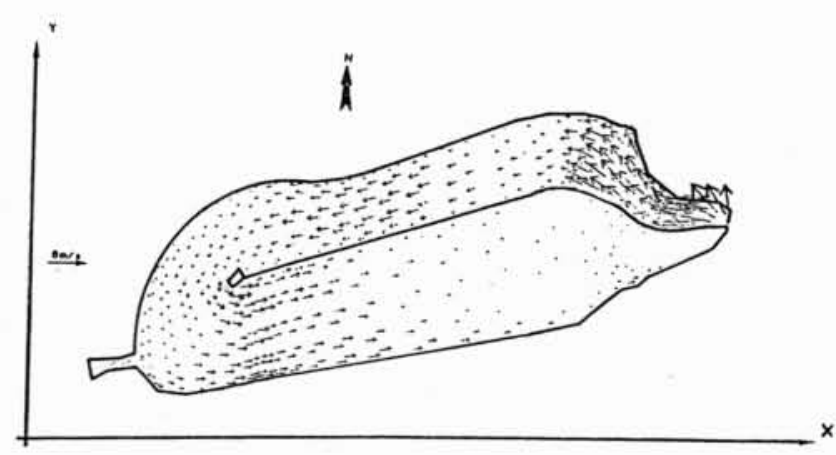

2. Champ de vitesse au cours de remplissage (vent Ouest).

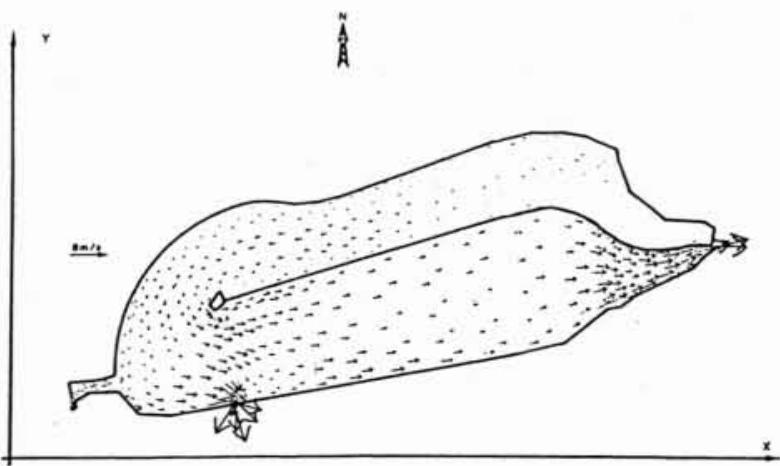

3. Champ de vitesse au cours de la vidange (vent Ouest). 


\section{Hydro-ecological model of the Tunis Lake}

This work concerns the setting up of a model to pre-determine changes in variable characteristics in the biotype and biocoenosis of algae in the Tunis Lake, in time and space. The model of water quality is coupled to a physical model simulating hydrodynamics, salinity and environment temperature. The model has been graded from measurements taken in situ before developments and valided from initial observations carried out after developments which consisted mainly in the construction of a dike and management of exchanges with the sea, and the deepening of certain zones.

Dans ce système d'équations $A$ désigne la section d'écoulement, définie par la berge, le fond et la digue, $h$ : hauteur d'eau, $Q$ : débit d'eau, $E_{h}$ : échanges d'eau avec le milieu extérieur, $g:$ accélération de la pesanteur, $Z_{f}$ : cote du fond, $\tau_{i}:$ frottement interfacial, $\tau_{p}:$ frottement au fond, $S$ : salinité, $E_{s}$ : taux d'échange de sel avec l'extérieur, $\Phi$ : flux d'énergie à l'interface eau-air, $E_{T}$ : taux d'échange thermique avec le milieu extérieur, $\rho$ : la masse volumique de l'eau, $C_{p}$ : chaleur spécifique de l'air à pression constante, $t$ : le temps et $X$ est l'axe principal de l'écoulement.

Trois types de conditions aux limites ont été prises en compte: frontière imperméable (flux nul), frontière libre (les échanges sont fonction du niveau de la marée) et frontière à débit imposé (centrale électrique) (fig. 2 et 3).

\section{Modèle écologique}

\subsection{Définition du modèle}

Avant les récents aménagements, les crises trophiques du lac de Tunis résultaient du rythme élevé d'eutrophisation mettant plus particulièrement en jeu les activités des macrophytes (Ulves) et du phytoplancton, ces deux populations algales pouvant entrer en compétition pour la lumière.

La modélisation adoptée propose une représentation de l'évolution de ces biomasses en relation avec le cycle de l'azote et du phosphore présents sous trois formes: organique particulaire, organique détritique, minéral dissous $\left(\mathrm{NH}_{4} \mathrm{~N}, \mathrm{NO}_{2}-\mathrm{N}, \mathrm{NO}_{3}-\mathrm{N}, \mathrm{PO}_{4}-\mathrm{P}\right)$.

Sur le schéma de la figure 4 les variables mises en jeu sont définies ainsi que les couplages pris en compte dans leur modélisation. Les formes minérales de l'azote et du phosphore sont les nutrients assimilés par le phytoplancton et les macrophytes et se transforment par conséquent en formes organiques particulaires.

La mortalité des macrophytes et du phytoplancton regénère de l'azote et du phosphore organique des détritus. Ces formes de nutrients sont décomposées en partie par les bactéries sous formes minérales. L'autre partie se dépose au fond du lac, il y a libération des nutrients sous forme minérale.

La libération de l'azote est attribuée pour l'essentiel à la décomposition de la matière organique dans les sédiments. Le taux de libération de l'azote minéral à partir des sédiments a été modélisé comme fonction de l'azote organique des sédiments, de la température et de la concentration d'oxygène près $\mathrm{du}$ fond. Ce modèle tient aussi compte de la compétition pour la lumière entre phytoplancton et ulves. Pendant les périodes d'éclosion du phytoplancton, l'eau devient très trouble, le coefficient d'extinction augmente, ce qui a pour effet de diminuer la pénétration de la lumière au fond du lac et d'empêcher toute croissance des ulves. Le modèle hydro-écologique inclut donc les variables d'état suivantes (fig. 4):

\begin{tabular}{|c|c|c|}
\hline $\begin{array}{l}\text { PHYT } \\
\text { Wu }\end{array}$ & $\begin{array}{l}\text { Biomasse du phytoplancton } \\
\text { Biomasse d'Ulva }\end{array}$ & $\begin{array}{l}\text { (mg/m' poids sec) } \\
\text { ( } / \mathrm{m}^{3} \text { poids humide) }\end{array}$ \\
\hline NS & Azote mintral dissous & (mg/m') \\
\hline NC & Azote du phytoplancton & $\left(\mathrm{mg} / \mathrm{m}^{3}\right)$ \\
\hline No & Azote des detritus & (mg/m') \\
\hline NSED & Azote du sediment & $\left(\mathrm{mg} / \mathrm{m}^{3}\right)$ \\
\hline PS & Phosphore mintral dissous & $\left(\mathrm{mg} / \mathrm{m}^{3}\right)$ \\
\hline PC & Phosphore des détritus & $\left(\mathrm{mg} / \mathrm{m}^{\prime}\right)$ \\
\hline PE & Phosphore \&changeable du sediment & (mg/m') \\
\hline $\mathrm{Pt}$ & Phosphore de l'eau interstitielle des sodiments & $\left(\mathrm{mg} / \mathrm{m}^{3}\right)$ \\
\hline C & Oxygène dissous & $(\mathrm{mg} /)$ \\
\hline D & Demande biochimique en oxygène & $(\mathrm{m} \Omega /)$ \\
\hline
\end{tabular}

L'évolution spatio-temporelle de ces variables est décrite par l'équation suivante:

$$
\frac{\partial C_{\alpha}}{\partial t}+\frac{\partial\left(Q C_{\alpha}\right)}{\partial X}=S_{\alpha}+D_{\text {fond }}+E_{\alpha}
$$

où $C_{\alpha}$ est la concentration du constituant $\alpha, S_{\mathrm{\alpha}}$ : taux de production ou de disparition du constituant $\alpha$ lié aux interactions écologíques. $D_{\text {fond }}$ : la diffusion de $C_{\mathrm{a}}$ à partir du fond et $E_{\alpha}$ est le taux d'échange avec l'extérieur.

Dans le terme $S_{\mathrm{a}}$ nous tenons compte des processus biologiques fondamentaux, à savoir :

Croissance du phytoplancton

Assimilation des nutrients par le phytoplancton

Mortalité du phytoplancton

Croissance des macrophytes

Mortalité des macrophytes

Compétition entre macrophytes et phytoplancton

Minéralisation des nutrients organiques dans les détritus en nutrients minéraux

Sédimentation des nutrients organiques des détritus

Libération de l'azote et du phosphore minéral à partir des sédiments.

Les lois des cinétiques utilisées sont celles de LEHMANN [3] complétées par des mesures in situ. Le cycle des nutrients (azote et phosphore) adopté dans ce 


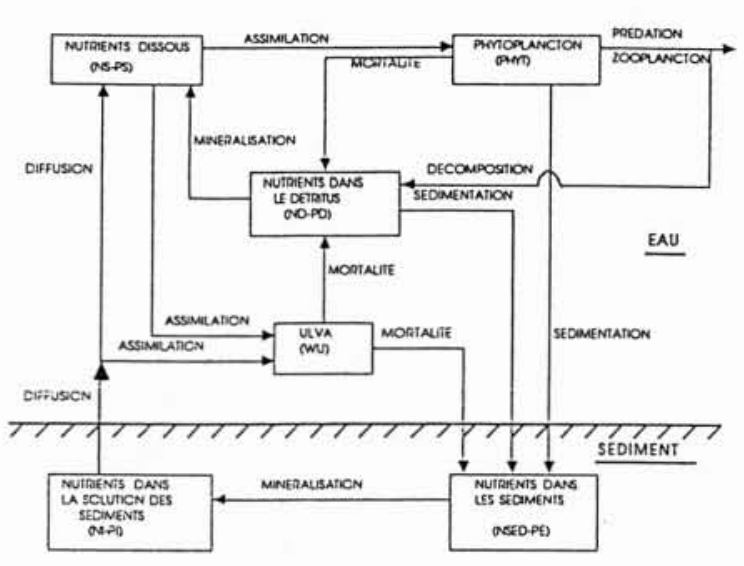

4. Schéma du cycle des nutrients (Azote et Phosphore).

modèle, et représenté sur la figure 1 est semblable à celui utilisé par JORGENSEN [4]. Ces lois et leurs différents coefficients sont présentés en annexe.

\section{Résolution numérique}

Pour la résolution numérique du système d'équations aux dérivées partielles (1) à (4) du modèle physique, nous avons adopté un schéma aux différences finies, centrée, explicite. Le pas de temps du modèle hydrodynamique est de $10 \mathrm{~min}$. Le rayonnement solaire est calculé toutes les $3 \mathrm{~h}$. La résolution des équations de la température et de la salinité a été effectuée avec un pas de temps de $12 \mathrm{~h}$. La résolution du système d'équations du modèle écologique a été réalisée à l'aide d'une méthode aux différences finies centrées avec un schéma implicite itératif et un pas de temps de $12 \mathrm{~h}$. Le lac de Tunis a été alors divisé en 5 zones considérées comme homogènes (fig. 4).

\section{Calage et validation du modèle}

Effectuées au cours de l'année 1984, des mesures mensuelles du phosphore total, de l'azote total, de la biomasse du phytoplancton, de la température et de l'oxygène dissous en 5 points du lac de Tunis ont été utilisées pour le calage du modèle. Le lac a été supposé homogène et une moyenne des mesures effectuées aux 5 points a été prise en compte. Les paramètres de calage (ci-contre) sont principalement le taux maximal de croissance du phytoplancton, les coefficients d'autoombrage des macrophytes et les taux de respiration des phytoplanctons et des macrophytes. Quelques résultats du calage sont représentés sur les figures 5 et 6 , qui montrent que le modèle rend bien compte de l'évolution annuelle de la biomasse du phytoplancton et de la température.

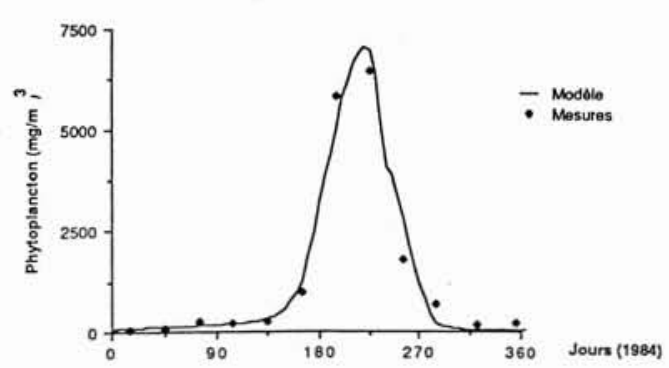

5. Calage du phytoplancton.

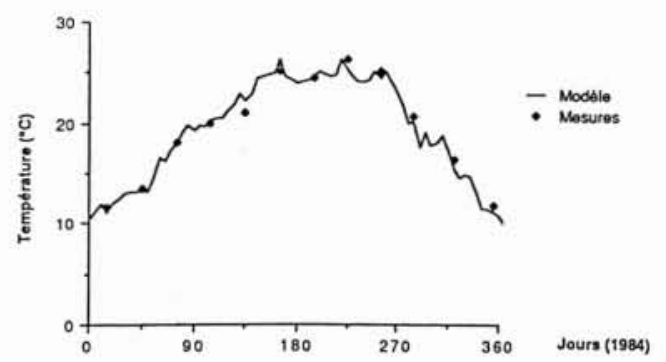

6. Calage de la température.

\begin{tabular}{|c|c|c|c|c|}
\hline \multicolumn{5}{|c|}{ Paramètres } \\
\hline Symbole & $\begin{array}{l}\text { Definition } \\
\text { Refierence }\end{array}$ & Unite & Valeur & \\
\hline APmax & Taux de croissance maximum du phytoplanction & $1 / \mathrm{j}$ & 1,5 & Calage \\
\hline AUmax & Taux de croissance maximum de rulva & $1 / 5$ & 0.4 & Zavodnic \\
\hline $\mathrm{K}_{\mathrm{p}}$ & Coefficient d'extinction specifique du phytoplancton & $\mathrm{m}^{2} / \mathrm{g}$ & 0,03 & Calcule \\
\hline A & Coefficient d'auto-ombrage relatif a rulva & - & 1,4 & Calage \\
\hline $\mathrm{Ke}$ & Coefficient d'extinction sptcifique de l'eau & $1 / \mathrm{m}$ & 0.1 & Calculé \\
\hline B & Coefficient d'auto-ombrage relatif a Yulva & - & 0,0018 & Calage \\
\hline DN & Taux de densification & $\mathrm{mgN} / \mathrm{m}^{2} / \mathrm{j}$ & 16 & Mesure \\
\hline $\mathrm{KN}$ & $\begin{array}{l}\text { Constante de demi-saturation pour lassimilation } \\
\text { de } \mathrm{N} \text { par le phyto }\end{array}$ & $\mathrm{mg} / \mathrm{m}^{3}$ & 200 & $\begin{array}{l}\text { Lehman } \\
\text { (79) }\end{array}$ \\
\hline KNR & Taux de liberation de IN & $\mathrm{mgN} / \mathrm{m}^{2} / \mathrm{j}$ & $2910^{-4}$ & Mesure \\
\hline KP & $\begin{array}{l}\text { Constante de demi-saturation pour lassimilation } \\
\text { du P par le phyto }\end{array}$ & $\mathrm{mg} / \mathrm{m}^{3}$ & 20 & $\begin{array}{l}\text { Lehman } \\
\text { (75) }\end{array}$ \\
\hline ко & $\begin{array}{l}\text { Constante prenant en compte linfluence de l'oxygetne } \\
\text { dissous }\end{array}$ & $\mathrm{mg} / \mathrm{l}$ & 1,7 & Calage \\
\hline $\mathrm{K}_{1}$ & Taux de degradation dans l'eau de la charge oxydable & $1 / \mathrm{j}$ & 0,65 & Calage \\
\hline $\mathrm{U}$ & Vitesse du vent & $\mathrm{m} / \mathrm{s}$ & - & Mesuret \\
\hline $\mathrm{K}_{3}$ & $\mathrm{Taux}_{\mathrm{d}} \mathrm{O}_{2}$ produit par $1 \mathrm{mg}$ de Chla & $\mathrm{mg} / \mathrm{mg}$ & 0,11 & Mesure \\
\hline $\mathrm{K}_{4}$ & Taux $\mathrm{dO}_{2}$ produit par $1 \mathrm{~g}$ dUlva & $\mathrm{mg} / \mathrm{g}$ & 0.9 & Mesure \\
\hline QNmin & $\begin{array}{l}\text { Quota cellulaire minimal de IN dans le phyto } \\
\text { Jorgensen (76) }\end{array}$ & $\mathrm{gN} / \mathrm{gPHYT}$ & 0.015 & \\
\hline QPmin & $\begin{array}{l}\text { Quota cellulaire minimal du P } \\
\text { Jorgensen (76) }\end{array}$ & ${ }_{\mathrm{gP}} / \mathrm{gPHYT}$ & 0,001 & \\
\hline Q & Quota cellulaire du phyto & $\mathrm{gN} / \mathrm{gPHYT}$ & - & - \\
\hline QN/max & $\begin{array}{l}\text { Quota cellulaire maximal de IN dans le phyto } \\
\text { Jorgensen (76) }\end{array}$ & $\mathrm{gN} / \mathrm{gPHYT}$ & 0.1 & \\
\hline $\mathrm{QP} / \max$ & $\begin{array}{l}\text { Quota cellulaire maximal du P dans le phyto } \\
\text { Jorgensen (76) }\end{array}$ & $\mathrm{gP} / \mathrm{gPHYT}$ & 0,013 & \\
\hline $\mathrm{KDN}_{20}$ & Taux de decomposition de IN organique à $20^{\circ} \mathrm{C}$ & $1 / j$ & 0,1 & Calage \\
\hline $\mathrm{KDP}_{20}$ & Taux de decomposition du P organique à $20^{\circ} \mathrm{C}$ & $1 / 5$ & 0,25 & Calage \\
\hline IOP group & Intensité optimale de production du phyto & $w / \mathrm{m}^{2}$ & 150 & $\mathrm{Lac}$ \\
\hline IOW group & Intensité optimale de production de rulva & $w / m^{2}$ & 350 & Lac \\
\hline H & Hauteur de la colonne d'eau & m & 1 & Mesure \\
\hline
\end{tabular}



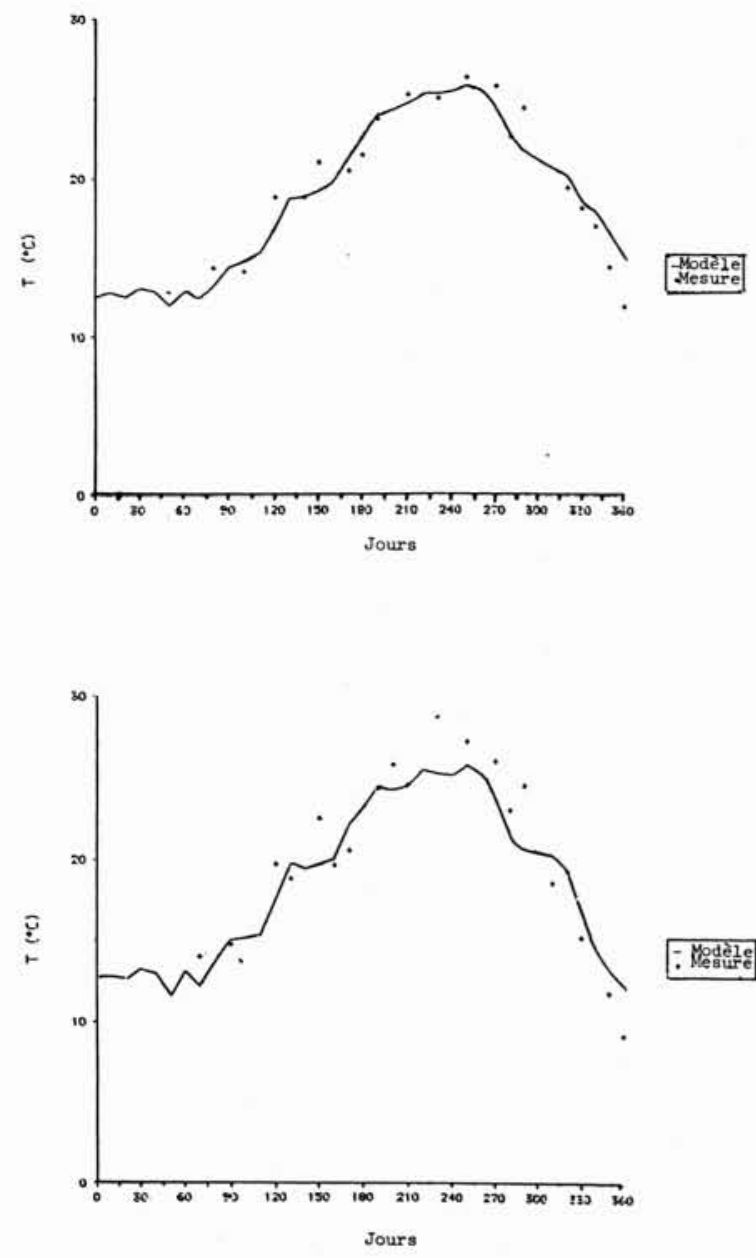

7. et 8. Validation température (zones 1 et 4).

Un contrôle systématique de la qualité des eaux a été effectué toutes les deux semaines, durant l'année 1988, après les aménagements, dans cinq stations du lac (au milieu de chaque zone). Ces données ont été utilisées pour la validation du modèle dans les cinq zones du lac de Tunis. Une partie des résultats de la validation est présentée sur les figures 7 et 8 (la température), 9 et 10 (la salinité), 11 et 12 (le phytoplancton) des zones 1 et 4 .

Nous remarquons que la qualité des eaux s'est considérablement améliorée dans tout le lac, ce qui est dû à l'augmentation des échanges avec la mer et au dragage des sédiments du fond (des zones 1, 2 et 3 ). Ainsi, ce modèle peut être utilisé pour prévoir l'évolution des différentes variables dans les cinq zones du lac de Tunis au cours des prochaines années (fig. 5 à 12).
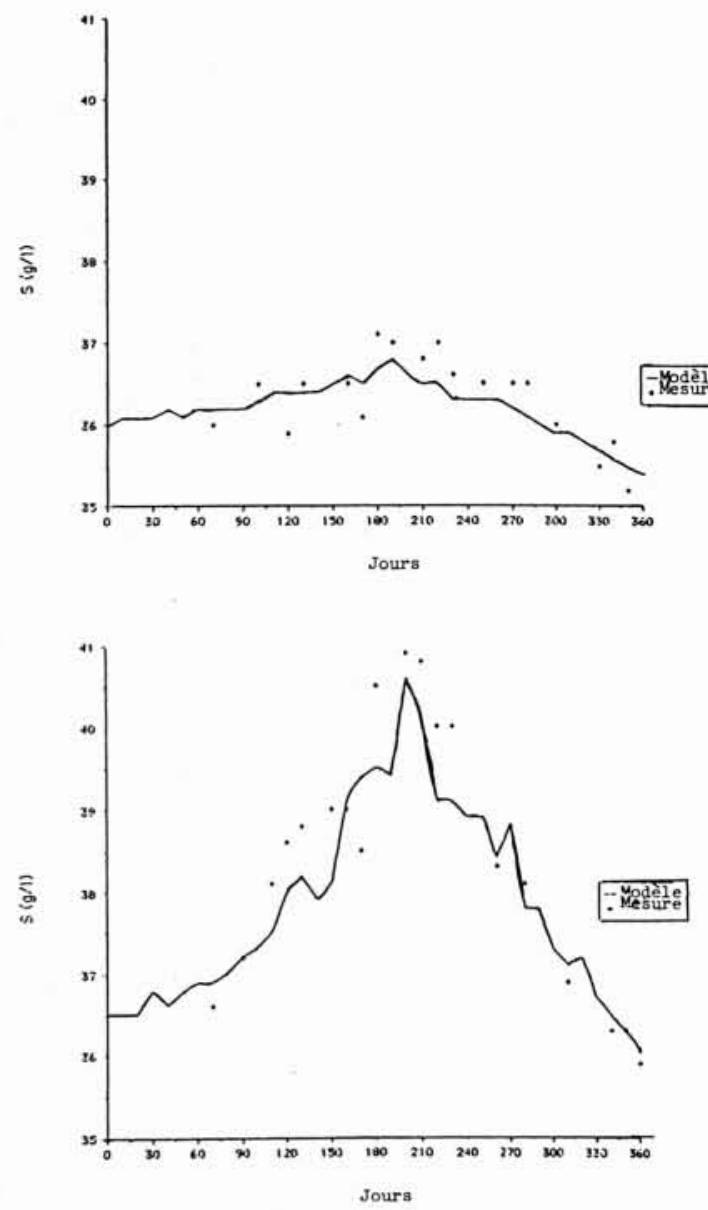

9. et 10. Validation salinité (zones 1 et 4).
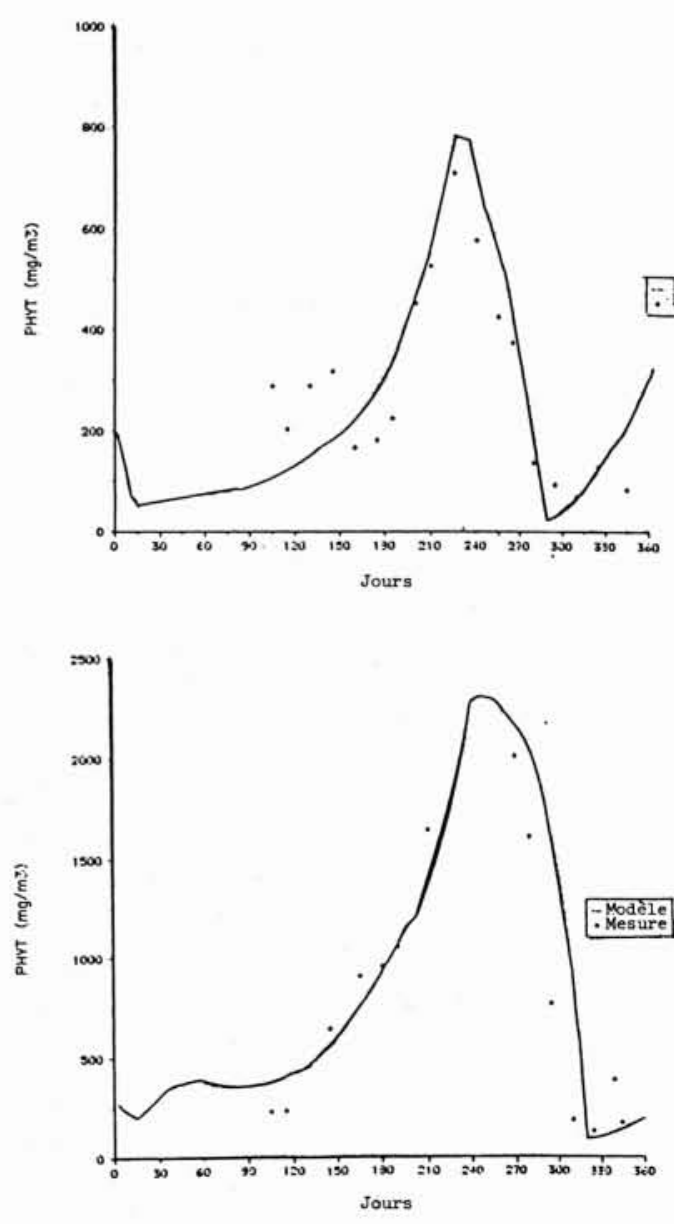

11. et 12. Validation Phyto (zones 1 et 4). 


\section{Conclusion}

Le modèle hydro-écologique de prédétermination des processus d'eutrophisation dans le lac de Tunis s'avère relativement performant: calé avant aménagement, il rend bien compte des évolutions de la qualité de l'eau du lac après les modifications importantes qu'a subies cet écosystème. Il reste cependant à l'améliorer pour introduire de nouvelles espèces algales dont le développement est attendu après les approfondissements réalisés. D'ores et déjà ce modèle constitue un outil relativement général pour l'étude des écosystèmes côtiers de faible profondeur. Présenté ici dans sa version ID qui est peu exigeante du point de vue des moyens de calcul et qui convenait très bien à l'étude du lac Nord de Tunis, il existe aussi en version $2 \mathrm{D}$ avec un traitement numérique en éléments finis. La mise au point d'une version 2D multicouche pour le traitement des zones côtières non homogène est en cours.

\section{Références}

[1] Ben Charrada R., Line A., Masbernat L., Moussa M. - Impact des aménagements sur l'hydrodynamique du lac de Tunis. Modẻle aux éléments finis. 2' Colloque Maghrébin sur les " Modèles numériques de l'Ingénieur ". Rabat (Maroc) novembre 1989.

[2] Moussa M. - Turbulence et circulation générées par le vent dans les svstèmes aquatiques peu profonds. Application du lac de Tunis. Thèse de Doctorat de l'INP de Toulouse, 1986.

[3] Lehmann J. T., Botkin D. B., Likens G. E. - The assumptions and rationales of a computer model of phytoplankton population dynamics. Limnol. Oceanog. 20, pp. 343-364, 1975.

[4] Jorgensen S. E. et al. - Validation of a prognosis based upon a eutrophication model. Ecol. Modelling 32, pp. 165182. 


\section{ANNEXE}

\section{Cinétiques adoptées}

1. $\frac{\mathrm{dPHYT}}{\mathrm{dt}}=(\mathrm{AP}-\mathrm{DP}) * \mathrm{PHYT}+\mathrm{E}_{\mathrm{PHYT}}$

2. $\frac{d W U}{d t}=(A U-M U) * W U$

3. $\frac{\mathrm{DNS}}{\mathrm{dt}}=\mathrm{KDN} * \mathrm{NO}-\mathrm{VN} * \mathrm{PHYT}-\mathrm{VNU}+\mathrm{NL}+\mathrm{DN}+\mathrm{ENS}$

4. $\frac{\mathrm{DNC}}{\mathrm{dt}}=\mathrm{VN} * \mathrm{PHYT}-\mathrm{DP} * \mathrm{NC}+\mathrm{ENC}$

5. $\frac{\mathrm{dNO}}{\mathrm{dt}}=\alpha * \mathrm{MU} * \mathrm{NU}+\mathrm{MP} * \mathrm{NC}-(\mathrm{KDN}+\mathrm{SD}) * \mathrm{NO}+\mathrm{ENO}$

6. $\frac{\mathrm{dNSED}}{\mathrm{dt}}=(1-\alpha) * \mathrm{MU} * \mathrm{NU}+\mathrm{SA} * \mathrm{NC}-\mathrm{NL} \cdot \mathrm{DN}$

7. $\frac{d P S}{d t}=K D P * P O-V P * P H Y T-V P U+Q P+E P S$

8. $\frac{\mathrm{dPC}}{\mathrm{dt}}=\mathrm{VP} * \mathrm{PHYT}-\mathrm{DP} * \mathrm{PC}+\mathrm{EPC}$

9. $\frac{d P O}{d t}=\alpha * M U * P U+M P * P C-(K D P+S D) * P O+E P O$

10. $\frac{\mathrm{dPE}}{\mathrm{dt}}=(1-\alpha) \mathrm{MU} \mathrm{PU}+\mathrm{SAPC}-\mathrm{KE} \mathrm{PE}$

11. $\frac{d P I}{d t}=K E \cdot P E \cdot Q P D F$

12. $\frac{d C}{d t}=K_{2}\left(C_{S}-C\right)+K_{3}(A P-M P) * P H Y T+K_{4}(A U-M U) W L-K_{1} D-B E N+E C$

13. $\frac{d D}{d t}=K_{3} S A * P H Y T-K_{1} D+E D$

\section{Facteurs limitants}

- Flum $=\frac{e}{\mathrm{KEH}} \cdot(\exp (-\mathrm{IZ} / \mathrm{IOP})-\exp (-\mathrm{IS} / \mathrm{IOP}))$

$-\mathrm{KE}=\mathrm{Kp} . \mathrm{PHYT}+\mathrm{Ke}$

$-\mathrm{Iz}=\mathrm{Is} . \exp (-\mathrm{KE} . \mathrm{H})$

- FT $=\exp (-2,3$ (T-Top) $1 / 5)$

$-\mathrm{FN} \mathrm{N}_{1} \mathrm{P}=\min (\mathrm{FN}, \mathrm{FP})$
$-\mathrm{ON}$ )

$-\mathrm{QNmin}=\mathrm{NC} / \mathrm{PH}$
$-\mathrm{OP}=\mathrm{PC} / \mathrm{PHYT}$

- QNmin $=$ NCmin/PHYT

- QPmin $=$ PCmin/PHYT

$-\mathrm{FN}=(\mathrm{QN}-\mathrm{QNmin}) /(\mathrm{QNmax}-\mathrm{QNmin})$

- FP $=(Q P-Q P \min ) /(Q P \max -Q P \min )$

$-\mathrm{GI}=\mathrm{IW} / \mathrm{NOW} \exp (1-\mathrm{IW} / \mathrm{IOW})$

$-\mathrm{IW}=\mathrm{IS} * \exp (-\mathrm{KE} . \mathrm{HW})$

$-\mathrm{GT}=\exp (-2,3(\mathrm{~T}-\mathrm{TOW}) / 1 / 5)$

$-\mathrm{GW}=\mathrm{A} \exp (-\mathrm{B}$. WU

$-\mathrm{FN}_{1}=\mathrm{QN \operatorname {max } - \mathrm { QN }}$

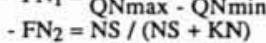

$\begin{aligned}-\mathrm{FN}_{2} & =\mathrm{NS} /(\mathrm{NS}+\mathrm{KN}) \\ -\mathrm{FP}_{1} & =\mathrm{QP} \max -\mathrm{QP}\end{aligned}$

QPmax-QPmi

$-\mathrm{FP}_{2}=\mathrm{PS} /(\mathrm{PS}+\mathrm{KP})$

- $\mathrm{FT}=(\mathrm{T} / \mathrm{TOW})^{1 / 2}$

- $\mathrm{FT}_{2}=(1,04)(\mathrm{T}-20)$

- $\mathrm{FT}_{3}=(1,09)(\mathrm{T}-20)$

$-\mathrm{FC}=\mathrm{KO} /(\mathrm{KO}+\mathrm{C})$

$-K_{2}=0,0002 * U^{2}+0,005$
Coefficients des cinétiques

.Taux de croissance du phyto

$\mathrm{AP}=\mathrm{APmax}$. Flum . FT . FNP

Taux de disparition du phyto

$\mathrm{DP}=\mathrm{MP}+\mathrm{SA}$

$\mathrm{MP}=\mathrm{K}_{4} \mathrm{~T}+\mathrm{D}_{1}$

$M P=K_{4} T+D_{1}+D_{2} \frac{\sin \left(d-d_{1}\right)}{\left(d_{2}-d_{1}\right)}$

pour $<d_{1}$ et $d>d_{2}$

pour $d_{1}<d<d_{2}$

Taux de croissance de l'ulva

$$
\mathrm{AU}=\mathrm{AU} \max . \mathrm{GI} \cdot \mathrm{GT} \cdot \mathrm{GW}
$$

.Taux de mortalité de l'ulva

$$
\mathrm{MU}=\mathrm{MU} \max . \mathrm{FT}_{1}
$$

Taux de décomposition de l'azote et du phosphore organique en minérale

$$
\mathrm{KDN}=\mathrm{KDP}_{20} \cdot \mathrm{FT}_{2}
$$$$
\mathrm{KDP}=\mathrm{KDP}_{20} \cdot \mathrm{FT}_{2}
$$

Taux d'assimilation de l'azote par le phytoplancton

$$
\mathrm{VN}=\mathrm{VN} \max : \mathrm{FN}_{1} \cdot \mathrm{FT}_{2}
$$

Taux d'assimilation du phosphore par le phytoplancton

$$
\mathrm{VP}=\mathrm{VP} \max \cdot \mathrm{FP}_{1} \cdot \mathrm{FP}_{2}
$$

Taux de libération de l'azote a partir des sédiments $\mathrm{QNDF}=\mathrm{KNR}$. NSED $, \mathrm{FT}_{3}, \mathrm{FC}$

Flux du phosphore minéral dissous d partir de leau interstitielle $\mathrm{QPDF}=\mathrm{KP}(\mathrm{Pi}-\mathrm{Ps}) \cdot \mathrm{FT}_{3} \cdot \mathrm{FC}$

\section{Fonctions de forçage}

QKH Débit joumalier entrant et sortant par Kheireddine $\left(\mathrm{m}^{3} / \mathrm{j}\right)$

QP Debit journalier entrant et sortant par la Pêcherie $\left(\mathrm{m}^{3} / \mathrm{j}\right)$

QCH Débit journalier sortant par Checkly $\left(\mathrm{m}^{3} / \mathrm{j}\right)$

QTM Débit journalier sortant par Tunis Marine $\left(\mathrm{m}^{3} / \mathrm{j}\right)$

QONAS Débit refoule par lONAS $\left(\mathrm{m}^{3} / \mathrm{j}\right)$

QSTEG Débit journalier pompé par la STEG $\left(\mathrm{m}^{3} / \mathrm{j}\right)$

Q Débit journalier $\left(\mathrm{m}^{3} / \mathrm{j}\right)$

T Température de l'eau $\left({ }^{\circ} \mathrm{C}\right)$

$\mathrm{S} \quad$ Salinité de leau $(\mathrm{g} / \mathrm{l})$

NSKH,NSST,NSP

Azote dissous entrant par Kheireddine, Steg et la Pêcherie (mg/m3)

$\mathrm{NCKH}, \mathrm{NCST}, \mathrm{NCP}$

Azote particulaire entrant par Kheireddine, STEG et la Pêcherie (mg/m3)

PSKH,PSST,PSP

Phosphore dissous entrant par Kheireddine, STEG et la Pêcherie

PCKH,PCST,PCP

Phosphore particulaire entrant par Kheireddine, STEG et la Pêcherie (mg/m3) NOKH,NOST,NOP

Azote organique des détritus entrant par Kheireddine, la STEG et la Pêcherie PHYTKH,PHMTK,PMTP

Phytoplancton entrant par Kheireddine, la STEG et la Pêcherie (mg/m3)

CKH,CP,CST Oxygène dissous entrant par Kheireddine, STEG et la Pêcherie (mg/) DKH,DP,DST D.B.O. des eaux entrant par Kheireddine, STEG et la Pêcherie (mg/) 